\title{
Increased Spinal Lumbar Fusion with Combined Magnetic Field (CMF) Bone Growth Stimulation
}

\author{
Rami Elsabeh ${ }^{1 *}$, Rae Stegall ${ }^{2}$ and John Abrahams ${ }^{1}$ \\ ${ }^{1}$ Brain and Spine Surgeons of New York, White Plains, NY, USA \\ ${ }^{2}$ Stegall Scientific, Austin, TX, USA
}

\begin{abstract}
The aim of this study was to assess the efficacy of bone growth stimulation (BGS), specifically Combined Magnetic Field (CMF) stimulation, in the recovery of lumbar spinal fusion patients. Electrical BGS devices are prescribed postoperatively to help with the healing of spinal fusion patients by promoting fusion. This retrospective cohort analysis examined 316 patients who received lumbar spinal fusion surgery at Brain \& Spine Surgeons of New York between the years 2013 and 2018. Patients were divided into 4 groups according to type of BGS used: Combined Magnetic Field (CMF), Capacitive Coupling (CC), Pulsed Electromagnetic Field (PEMF) and No Stimulation (No-Stim). The average time-to-fusion for the CMF group was 5.5 months compared to 8.1 months for the No-Stim group $(p=0.003)$. Patients receiving CMF stimulation fused 75 days faster than patients receiving PEMF stimulation $(p=0.02)$ and 42 days faster than patients receiving CC stimulation. Smokers and spinal stenosis patients using the CMF stimulator also fused the fastest in their respective subgroups. The CMF group displayed the highest overall fusion rate at $95.7 \%$ and had the highest fusion rates for all patient subgroups studied. Electrical bone growth stimulation in general resulted in faster fusion times and greater fusion rates.
\end{abstract}

\section{Keywords}

Lumbar, Spine, Spinal, Spinal fusion, CMF, Combined magnetic field, Electrical bone growth stimulation

\section{Introduction}

Lumbar spinal fusions are being performed at increasing frequency, with almost two-hundred-thousand cases performed in the United States in 2015, a 62.3\% increase in volume since 2004 . Fusion procedures performed for disc degeneration, disc herniation and spinal stenosis accounted for $42 \%$ of the total elective lumbar cases in 2015. Rapidly rising costs of spinal surgery have resulted in over $\$ 10.2$ billion dollars in yearly hospital costs for these procedures, amounting to more than $\$ 50,000$ per patient admission [1], a $177 \%$ increase since 2004 . The mean age of lumbar fusion patients continues to rise. The mean age in 2001 was 52 and this rose to 54.6 in 2004, 57 in 2013 and 59.9 in 2015 [1,2]. This rising average age has been linked to a greater comorbidity burden and an increase in risks for perioperative complication outcomes [2]. Patients with degenerative spinal conditions may require lumbar fusion if they do not respond to non-operative treatment and have abnormal and/or excessive motion at a vertebral segment resulting in severe pain and/or inability to function. Other conditions that may require fusion include a weak or unstable spine, possibly caused by infections or tumors, fractures, scoliosis and spinal deformities.

Spinal fusion surgery is designed to decrease pain ge- nerated from the joints by stopping the motion at a painful vertebral segment. The approach to fusion includes adding a bone graft or bone graft substitute to the spine segment which, through a biological response, causes bone growth and fusion between the vertebra, resulting in the formation of a single, solid bone. Historically a patient's hip bone, the iliac crest, was harvested for spinal fusions but due its donor site morbidity physicians have shifted toward using allograft, non-iliac crest autograft and bone graft substitutes [3].

Failed solid bony fusion, known as pseudarthrosis, is a serious, prevalent and costly complication of spinal fusion procedures [4]. It is known to cause persistent or recurrent pain and disability, as shown by Kornblum, et al. [5] in a prospective, randomized study focused on the long-term influence of pseudoarthrosis on clinical outcomes in patients who

*Corresponding author: Rami Elsabeh, Brain and Spine Surgeons of New York, White Plains, NY, USA, Tel: +1-914-948-8448

Accepted: January 06, 2020

Published online: January 08, 2020

Citation: Elsabeh R, Stegall R, Abrahams J (2020) Increased Spinal Lumbar Fusion with Combined Magnetic Field (CMF) Bone Growth Stimulation. J Orthop Surg Tech 3(1):84-91 
received posterior lumbar fusion. It was found that patients who presented with pseudoarthrosis were significantly less likely to have a clinical outcome of excellent to good and had significantly higher back and lower limb pain than patients without pseudoarthrosis. Lumbar pseudarthrosis causes quality of life challenges in the patient's physical and mental experience and can result in severe morbidity [6].

Pseudoarthrosis is often suspected when a fusion patient presents with recurrent or neurological symptoms during long-term follow-up. A significant rate of pseudoarthrosis has been reported in the literature ranging from 15 to $43 \%[7,8]$, and rates at 1-year can be as high as $35 \%$ [9]. The prevalent rate of symptomatic pseudoarthrosis in lumbar spinal fusions is $10-15 \%[10,11]$. This high incidence rate is significant as pseudoarthrosis is one of the most common indications for repeat surgery, and one of the most common revision surgery indications is when symptomatic non-fusion occurs [12]. There are certain risk factors associated with pseudoarthrosis which include smoking, obesity, diabetes, multilevel fusions, previously failed fusion, and osteoporosis $[13,14]$.

The best treatment for pseudarthrosis is to prevent it from occurring after the initial operation [13]. Because of the complications caused by pseudoarthrosis and the fact that fusion has been shown to be positively correlated to improved clinical and functional outcomes [1], achieving a solid fusion is the ultimate goal of spinal fusion procedures. Given the number of patients undergoing lumbar spinal fusion procedures and the percentage of failed fusions, consideration of adjunctive therapies to further enhance the probability of fusion is warranted [15].

One of these adjunctive therapies is electrical stimulation. The clinical benefits of electrical stimulation as adjuncts to spinal fusions have become increasingly recognized over the last 30 years. Scientific studies have better defined their mechanisms of action, thus supporting the validity of these treatments. Bone formation and fusion involves a complex series of cellular interactions and molecular pathways [16]. Learning how to heal injured bone and induce and accelerate bone growth and repair has led to the creation of bone growth stimulator (BGS) devices which employ various technologies that revolve around electrical stimulation. These devices are prescribed post-operatively to help mimic the body's natural healing environment by generating electric fields that activate the body's internal repair mechanism which promotes bone growth [16].

Three types of electrical stimulation technologies have been FDA approved for clinical use. These technologies are direct current (DC), capacitive coupling (CC), and inductive coupling (IC) such as pulsed electromagnetic fields (PEMF) and combined magnetic fields (CMF) [17].

Previous generations of electrical stimulation technologies like DC have been shown to be effective fusion adjuncts, but these technologies are invasive, which has led to the more frequent use of newer, external non-invasive technologies such as CC and IC. An external electrical stimulation device is worn for a specified number of hours a day for a period of approximately 3 to 9 months following the surgery. With the CC device, metal electrodes with conductive gel are applied to the skin and are connected to an alternating current signal generator. With the IC device, an electromagnetic field, either CMF or PEMF, is created by an electrical current and transmitted through treatment coils that are placed into a brace and externally onto the fusion site. CMF treatment can promote bone cell proliferation through the release of IGF-II, which acts as a feedback loop [18]. Modelling for both the interbody and posterolateral fusion models showed that CMF therapy has a robust $100 \%$ coverage of the $L 1$ to $L 5$ vertebrae and all the intervertebral disc spaces [15]. Both CMF and PEMF involve the use of low energy, low-frequency magnetic field pulses, however CMF treatment differs from PEMF treatment by using a combination of static and alternating dynamic electromagnetic fields instead of one static electromagnetic field that pulses on or off.

External non-invasive therapeutic electrical BGSs have been shown to be successful when evaluated as an adjunct to spinal fusion surgery to enhance the chances of obtaining a solid spinal fusion or to treat a failed fusion. However, there is still controversy regarding which stimulation technology is most effective under certain clinical scenarios $[9,19]$. The main focus of this retrospective study is to evaluate the relationship between using a BGS, especially the CMF SpinaLogic $B G S$, and the success of bone fusion after lumbar spinal surgery. This study provides an overview of external BGS stimulation as an adjunct to spinal fusion and summarizes indications while focusing on the CMF stimulation technology.

\section{Materials and Methods}

\section{Study design and patients}

This study was a retrospective review of lumbar spinal fusion surgeries performed between 2013 and 2018 by 11 surgeons at Brain and Spine Surgeons of New York (BSSNY). The data was prospectively collected in BSSNY's Centricity PMS system through chart abstraction.

It was an all-comers study with the one inclusion criteria being that there was sufficient information in the patient's chart to assess the patient's fusion status. There were no exclusion criteria. All lumbar fusion procedures performed used instrumentation. The statistical tests utilized were ANOVA, Chi-squared test and Tukey's HSD range test.

There was a total of 316 patients examined with an average follow-up of 6 months. Patients were stratified into one of four cohorts. In three of the cohorts a particular non-invasive BGS was used as an adjunct to fusion (CMF, PEMF or CC) and in the fourth cohort no stimulation (NoStim) was used. The PEMF device was worn for 8 hours a day and the CC device was worn for 24 hours a day. The CMF SpinaLogic ${ }^{\circledR}$ Bone Growth Stimulator (DJO Global, Dallas, Texas) was worn by patients for 30 minutes a day. This stimulator is shaped to accommodate spine curvature and promotes fusion at one or two levels.

A stimulator was used in $72 \%(226 / 316)$ of patients and $28 \%(90 / 316)$ received no stimulation. The stimulator brand 
Citation: Elsabeh R, Stegall R, Abrahams J (2020) Increased Spinal Lumbar Fusion with Combined Magnetic Field (CMF) Bone Growth Stimulation. J Orthop Surg Tech 3(1):84-91

Table 1: Patient demographics.

\begin{tabular}{|c|c|c|c|c|c|c|}
\hline Patient Demographics & & Distribution & Patient Cour & & & \\
\hline Group (\% patients unknown) & Group & No-Stim & CMF & PEMF & CC & Total \\
\hline & & $n=90$ & $n=115$ & $n=54$ & $n=57$ & $n=316$ \\
\hline Gender & Female & $54.4 \%(49)$ & $40.9 \%(47)$ & $48.1 \%(26)$ & $57.9 \%(33)$ & $49.1 \%(155)$ \\
\hline & Male & $45.6 \%(41)$ & $59.1 \%(68)$ & $51.9 \%(28)$ & $42.1 \%(24)$ & $50.9 \%(161)$ \\
\hline Smoking History (8.5\%) & Current/Former & $52.3 \%(47)$ & $57.4 \%(66)$ & $59.3 \%(32)$ & $45.6 \%(26)$ & $54 \%(171)$ \\
\hline & No & $29.8 \%(26)$ & $40 \%(46)$ & $38.9 \%(21)$ & $43.9 \%(25)$ & $37.3 \%(118)$ \\
\hline Diabetes History (11.7\%) & Yes & $14.4 \%(13)$ & $16.5 \%(19)$ & $7.4 \%(4)$ & $10.5 \%(6)$ & $13.3 \%(42)$ \\
\hline & No & $70 \%(63)$ & $73 \%(84)$ & $88.9 \%(48)$ & $73.7 \%(42)$ & $75 \%(237)$ \\
\hline Age & Age $<55$ & $55.6 \%(50)$ & $43.5 \%(50)$ & $33.3 \%(18)$ & $43.9 \%(25)$ & $45.3 \%(143)$ \\
\hline & Age $\geq 55$ & $44.4 \%(40)$ & $56.5 \%(65)$ & $66.7 \%(36)$ & $56.1 \%(32)$ & $54.7 \%(173)$ \\
\hline & Age $61-70$ & $23.3 \%(21)$ & $29.6 \%(34)$ & $37 \%(20)$ & $28.1 \%(16)$ & $28.8 \%(91)$ \\
\hline BMI (11.1\%) & $\leq 29$ & $45.6 \%(41)$ & $54.8 \%(63)$ & $50 \%(27)$ & $45.6 \%(26)$ & $49.7 \%(157)$ \\
\hline & $\geq 30$ & $34.4 \%(31)$ & $42.6 \%(49)$ & $44.4 \%(24)$ & $35.1 \%(20)$ & $39.2 \%(124)$ \\
\hline Exercise History (24.1\%) & Never & $13.3 \%(12)$ & $17.4 \%(20)$ & $18.5 \%(10)$ & $10.5 \%(6)$ & $15.2 \%(48)$ \\
\hline & Rarely & $16.7 \%(15)$ & $35.6 \%(41)$ & $29.6 \%(16)$ & $40 \%(23)$ & $30.1 \%(95)$ \\
\hline & Weekly & $18.8 \%(17)$ & $27.8 \%(32)$ & $33.3 \%(18)$ & $12.3 \%(7)$ & $23.4 \%(74)$ \\
\hline Previous Spine Surgery & 0 & $48.9 \%(44)$ & $59.1 \%(68)$ & $57.4 \%(31)$ & $43.9 \%(25)$ & $53.2 \%(168)$ \\
\hline & 1 & $24.4 \%(22)$ & $25.2 \%(29)$ & $25.9 \%(14)$ & $35.1 \%(20)$ & $26.9 \%(85)$ \\
\hline & 2 & $18.9 \%(17)$ & $11.3 \%(13)$ & $9.3 \%(5)$ & $8.8 \%(5)$ & $12.7 \%(40)$ \\
\hline & $3+$ & $2.2 \%(2)$ & $3.5 \%(4)$ & $5.6 \%(3)$ & $5.3 \%(3)$ & $3.8 \%(12)$ \\
\hline
\end{tabular}

Table 2: Diagnosis demographics.

\begin{tabular}{|l|l|}
\hline Diagnosis & $\begin{array}{l}\text { Distribution \% } \\
\text { (Patient Count) } \mathbf{n}=\mathbf{3 1 6}\end{array}$ \\
\hline Adjacent Segment Disease & $1 \%(4)$ \\
\hline Degenerative Disc Disease & $28 \%(88)$ \\
\hline Disc Herniation & $36 \%(114)$ \\
\hline Instability & $4 \%(14)$ \\
\hline Prior Discectomy & $2 \%(7)$ \\
\hline Prior Fusion Surgery & $5 \%(17)$ \\
\hline Pseudoarthrosis & $2 \%(5)$ \\
\hline Spondylolisthesis & $27 \%(86)$ \\
\hline Stenosis & $47 \%(147)$ \\
\hline
\end{tabular}

distribution percentage of the 226 patients using a BGS was as follows: CMF with $51 \%(115)$, PEMF with $24 \%(54)$, and CC with $25 \%$ (57). Patient demographic and baseline characteristic data is presented in Table 1. Table 2 shows the diagnosis demographics and Table 3 displays the surgical demographics.

The risk profile for the total patient population consisted of $55 \%$ aged 55 and over, $39 \%$ with a BMI of 30 or greater, $54 \%$ who are current or former smokers, $1.6 \%$ with osteopenia, $4.4 \%$ with osteoporosis and $13 \%$ with diabetes. The CMF patient risk profile was as follows: $57 \%$ aged 55 and over, $43 \%$ with a BMI of 30 and above, $57 \%$ who are current or former smokers, $3 \%$ with osteopenia, $5 \%$ with osteoporosis, and $17 \%$ with diabetes.

Fusion Status was determined by both radiographical and clinical evidence. The patient was documented as "Fused" if this status was explicitly stated in imaging reports and/or if the trusted surgeon's post-operative notes stated that the patient was doing clinically well and did not need further follow-up. One of the reasons patients do not follow up after surgery is because they are recovering well and feel that they no longer need medical care. Typically, patients would return for a follow-up visit if they were experiencing recurrent or new symptoms. If the imaging reports clearly articulated pseudoarthrosis, the patient's status was labeled as "Not Fused", and $100 \%$ of the not-fused patients were deemed so by radiography.

\section{Results}

Fusion rate and fusion time (time-to-fusion) are the two outcome measures used in this study to assess treatment success of patients receiving lumbar fusion surgery. Fusion rate was determined according to the definition outlined in the Methods/Materials section. Fusion time was treated as a continuous variable. Overall fusion rate and fusion time are reviewed, as well as the fusion rate and fusion time of specific patient subgroups. Due to a lack of consistent documentation in the patients' charts, for several of the patient factors there is no information available for the PEMF and CC patient groups. Below we summarize the findings for each outcome measured and compare results among the patient groups (when information is available). 
Citation: Elsabeh R, Stegall R, Abrahams J (2020) Increased Spinal Lumbar Fusion with Combined Magnetic Field (CMF) Bone Growth Stimulation. J Orthop Surg Tech 3(1):84-91

Table 3: Surgical demographics.

\begin{tabular}{|c|c|c|c|c|c|c|}
\hline \multirow{2}{*}{$\begin{array}{l}\text { Surgical Demographics } \\
\begin{array}{l}\text { Group (\% patients } \\
\text { unknown) }\end{array} \\
\end{array}$} & \multirow[b]{2}{*}{ Group } & \multicolumn{4}{|c|}{ Distribution \% (Patient Count/Total) } & \multirow[b]{2}{*}{ Total } \\
\hline & & No-Stim & CMF & PEMF & CC & \\
\hline \multirow[t]{2}{*}{ Operative Distribution } & Minimally Invasive & $50 \%(17 / 34)$ & $26 \%(9 / 34)$ & $9 \%(3 / 34)$ & $15 \%(5 / 34)$ & $11 \%(34 / 316)$ \\
\hline & Open & $26 \%(73 / 282)$ & $38 \%(106 / 282)$ & $18 \%(51 / 282)$ & $18 \%(52 / 282)$ & $89 \%(282 / 316)$ \\
\hline \multirow[t]{2}{*}{ Level Distribution } & Single-Level & $57 \%(51 / 90)$ & $53 \%(61 / 115)$ & N/A & N/A & $55 \%(112 / 205)$ \\
\hline & Multi-Level & $43 \%(39 / 90)$ & $47 \%(54 / 115)$ & N/A & N/A & $45 \%(93 / 205)$ \\
\hline \multirow[t]{4}{*}{ Surgical Approach (1\%) } & Posterior & $29 \%(63 / 221)$ & $34 \%(74 / 221)$ & $18 \%(40 / 221)$ & $20 \%(44 / 221)$ & $70 \%(221 / 316)$ \\
\hline & Anterior & $30 \%(8 / 27)$ & $33 \%(9 / 27)$ & $22 \%(6 / 27)$ & $15 \%(4 / 27)$ & $9 \%(27 / 316)$ \\
\hline & Lateral & $26 \%(12 / 46)$ & $57 \%(26 / 46)$ & $9 \%(4 / 46)$ & $9 \%(4 / 46)$ & $15 \%(46 / 316)$ \\
\hline & Posterior and Anterior & $20 \%(3 / 15)$ & $33 \%(5 / 15)$ & $20 \%(3 / 15)$ & $27 \%(4 / 15)$ & $5 \%(15 / 316)$ \\
\hline \multirow[t]{3}{*}{ Bone Graft (2\%) } & Allograft & $24 \%(15 / 62)$ & $40 \%(25 / 62)$ & $15 \%(9 / 62)$ & $21 \%(13 / 62)$ & $20 \%(62 / 316)$ \\
\hline & Autograft & $29 \%(36 / 123)$ & $33 \%(40 / 123)$ & $24 \%(30 / 123)$ & $14 \%(17 / 123)$ & $39 \%(123 / 316)$ \\
\hline & Allograft + Autograft & $27 \%(33 / 122)$ & $39 \%(48 / 122)$ & $12 \%(15 / 122)$ & $21 \%(26 / 122)$ & $39 \%(122 / 316)$ \\
\hline
\end{tabular}

Table 4: Overall fusion rate and time-to-fusion.

\begin{tabular}{|l|l|l|l|}
\hline Device & Fused/Total Patient Count & Overall Fusion Rate $\%$ & Overall Fusion Time: Mean Days-to-Fusion \\
\hline No-Stim & $83 / 90$ & 92.2 & 244 \\
\hline CMF & $110 / 115$ & 95.7 & $166^{*}$ \\
\hline PEMF & $50 / 54$ & 92.6 & 241 \\
\hline CC & $54 / 57$ & 94.7 & 208 \\
\hline
\end{tabular}

*Statistically Significant, $p=0.002$.

Table 5: Fusion rates by high risk factors.

\begin{tabular}{|l|l|l|l|l|}
\hline High Risk Factors & Device Fusion Rates (Patient Count Fused/Total) \% \\
\hline & No-Stim & CMF & PEMF & CC \\
\hline Smokers-Overall & $89.4 \%(42 / 47)$ & $92.4 \%(61 / 66)$ & $87.5 \%(28 / 32)$ & $92.3 \%(24 / 26)$ \\
\hline Age 61-70 & $90.5 \%(19 / 21)$ & $100 \%(34 / 34)$ & $95 \%(19 / 20)$ & $93.8 \%(15 / 16)$ \\
\hline Previous Spine Surgery & $92.7 \%(38 / 41)$ & $95.7 \%(44 / 46)$ & $90.9 \%(20 / 22)$ & $92.9 \%(26 / 28)$ \\
\hline
\end{tabular}

\section{Fusion rate}

Overall fusion success rates were higher in patients receiving electrical stimulation, and CMF stimulation resulted in the highest overall fusion rate at $95.7 \%(110 / 115)$ with the No-Stimulator group having the lowest fusion rate at $92.2 \%$ (83/90) (Table 4).

The CMF stimulator group also had the highest fusion rate for patients with known risk factors for lumbar fusion surgery, specifically smokers (current or former), patients between the ages of 61-70, and patients with a history of previous spine surgery. For overall smokers, 92.4\% (61/66) fused when using the CMF stimulator compared to $92.3 \%$ $(24 / 26)$ when using the CC stimulator, $89.4 \%$ (42/47) when using no stimulator, and $87.5 \%(28 / 32)$ when using the PEMF stimulator. Regarding patients between the ages of $61-70$, the CMF stimulator group had a $100 \%(34 / 34)$ fusion rate and the fusion rates for the PEMF, CC and NoStim groups were 95\% (19/20), 93.8\% (15/16) and 90.5\% $(19 / 21)$, respectively. For these patients, fusion success rates were higher in those receiving electrical stimulation. Approximately ninety-six percent of patients (44/46) who received CMF stimulation and had a history of previous spine surgery fused. This was the highest fusion rate among all cohorts for this patient subgroup. The CC NoStim and PEMF cohorts had fusion rates of $92.9 \%(26 / 28)$, $92.7 \%(38 / 41)$, and $90.9 \%(20 / 22)$, respectively (Table 5$)$.

Other patient factors investigated include gender, diagnosis, return-to-work ability, and pre-operative exercise history. When compared to the other stimulator groups and the no-stimulator group, the CMF group had the greatest fusion rates for patients who are female and patients who have a primary diagnosis of spinal stenosis; fusion rates for the CMF group were $95.7 \%$ (45/47) and $100 \%$ $(28 / 28)$, respectively. Female patients who received no stimulation and patients with a primary diagnosis of spinal stenosis who received no stimulation displayed the lowest fusion rates in their respective subgroups at $87.8 \%(43 / 49)$ and $88.9 \%$ (16/18) (Table 6 and Table 7).

Fusion data for patients who returned to work and 
Citation: Elsabeh R, Stegall R, Abrahams J (2020) Increased Spinal Lumbar Fusion with Combined Magnetic Field (CMF) Bone Growth Stimulation. J Orthop Surg Tech 3(1):84-91

Table 6: Fusion rate by patient factors.

\begin{tabular}{|l|l|l|l|l|}
\hline Other Patient Factors & \multicolumn{4}{ll}{ Device Fusion Rates (Patient Count Fused/Total) \% } \\
\hline & No-Stim & CMF & PEMF & CC \\
\hline Spinal Stenosis & $88.9 \%(16 / 18)$ & $100 \%(28 / 28)$ & $92.9 \%(13 / 14)$ & $90.9 \%(10 / 11)$ \\
\hline Female & $87.8 \%(43 / 49)$ & $95.7 \%(45 / 47)$ & $88.5 \%(23 / 26)$ & $93.9 \%(31 / 33)$ \\
\hline Never Exercise & $92 \%(11 / 12)$ & $100 \%(20 / 20)$ & N/A & N/A \\
\hline Rarely Exercise & $80 \%(12 / 15)$ & $95.1 \%(39 / 41)$ & N/A & N/A \\
\hline Ability to Return to Work Overall & $84 \%(16 / 19)$ & $100 \%(26 / 26)$ & N/A & N/A \\
\hline
\end{tabular}

Table 7: Fusion time by patient factors.

\begin{tabular}{|l|l|l|l|l|}
\hline Patient Factors & \multicolumn{4}{l|}{ Device Fusion Time - Mean Days-to-Fusion (Patient Count) } \\
\hline & No-Stim & CMF & PEMF & CC \\
\hline Smokers Overall & $242(42)$ & $175(61)$ & $219(28)$ & $216(24)$ \\
\hline Spinal Stenosis & $267(16)$ & $152^{*}(28)$ & $356(13)$ & $203(10)$ \\
\hline
\end{tabular}

*Statistically Significant, $p<0.001$.

Table 8: Overall fusion time ANOVA.

\begin{tabular}{|l|l|l|l|l|l|}
\hline Statistical Comparison & ANOVA & dF & Mean Square & F & Sig. \\
\hline & Sum of Squares & dF & 5.178 & 0.002 \\
\hline Between Groups & 350519.479 & 3 & 116839.826 & \\
\hline Within Groups & 6588501.275 & 292 & 22563.361 & \\
\hline Total & 6939020.753 & 295 & & \\
\hline
\end{tabular}

Significant difference, $p=0.002$.

Table 9: Overall fusion time Tukey HSD.

\begin{tabular}{|c|c|c|}
\hline \multicolumn{3}{|l|}{$\begin{array}{l}\text { Tukey HSD } \\
\text { Multiple Comparisons }\end{array}$} \\
\hline \multicolumn{3}{|c|}{ Dependent Variable: Time to Fusion } \\
\hline (I) Brand of stimulator & (J) Brand of stimulator & Sig. \\
\hline \multirow[t]{3}{*}{ No-Stim } & PEMF & 1.000 \\
\hline & CMF & 0.003 \\
\hline & $\mathrm{CC}$ & 0.525 \\
\hline \multirow[t]{3}{*}{ PEMF } & No-Stim & 1.000 \\
\hline & CMF & 0.020 \\
\hline & $\mathrm{CC}$ & 0.676 \\
\hline \multirow[t]{3}{*}{ CMF } & No-Stim & 0.003 \\
\hline & PEMF & 0.020 \\
\hline & $\mathrm{CC}$ & 0.343 \\
\hline \multirow[t]{3}{*}{ CC } & No-Stim & 0.525 \\
\hline & PEMF & 0.676 \\
\hline & CMF & 0.343 \\
\hline
\end{tabular}

CMF was significantly lower than No-Stim, $p=0.003$; CMF was significantly lower than PEMF, $p=0.02$.

those with known pre-operative exercise history information was only available for the CMF and No-Stim groups. Patients in the CMF stimulator group who returned to work fused at $100 \%(26 / 26), 16 \%$ more than patients in the
No-Stim group. For those patients stating that they never or rarely exercised, ones that used the CMF stimulator fused more than those using no stimulator, with CMF fusion rates of $100 \%(20 / 20)$ and $95.1 \%(39 / 41)$, respectively (Table 6).

\section{Fusion time}

Overall, patients in the electrical stimulation groups had shorter times-to-fusion (fused faster) than patients in the group receiving no electrical stimulation. The CMF stimulator group had a mean fusion time of 166 days (SD 112.21), or 5.5 months, shortest among any of the patient groups. The mean fusion time for the CC, PEMF and No-Stim groups were 208 days (SD 136.11), or 7 months, 241 days (SD 175.68), or 8 months, and 244 days (SD 183.30), or 8.1 months, respectively (Table 4). ANOVA tests revealed a statistically significant difference between the groups ( $p=0.002$ ) (Table 8), and Tukey's HSD tests showed that patients using the CMF stimulator fused significantly faster (time-to-fusion was significantly less) than patients using no stimulator $(p=0.003)$ and patients using the PEMF stimulator $(p=0.02$ ) (Table 9).

This held true for patients with a primary diagnosis of spinal stenosis. Patients who had this stenosis diagnosis and received CMF stimulation had a mean fusion time of 152 days (SD 99.28), which was significantly shorter than the mean fusion times of stenosis patients receiving no stimulation ( $p=$ 0.061 ) and PEMF stimulation ( $p<0.001$ ) by 115 and 204 days, respectfully. The CMF group also had a shorter fusion time when compared to the CC group, although this difference was 
Citation: Elsabeh R, Stegall R, Abrahams J (2020) Increased Spinal Lumbar Fusion with Combined Magnetic Field (CMF) Bone Growth Stimulation. J Orthop Surg Tech 3(1):84-91

Table 10: Spinal stenosis fusion time ANOVA.

\begin{tabular}{|l|l|l|l|l|l|}
\hline Statistical Comparison & ANOVA & dF & Mean Square & F & Sig. \\
\hline & Sum of Squares & dF & 6.617 & 0.001 \\
\hline Between Groups & 402515.568 & 3 & 134171.856 & \\
\hline Within Groups & 1277351.208 & 63 & 20275.416 & \\
\hline Total & 1679866.776 & 66 & & \\
\hline
\end{tabular}

Significant main effect, $p<0.001$.

Table 11: Spinal stenosis fusion time Tukey HSD.

\begin{tabular}{|c|c|c|}
\hline \multicolumn{3}{|l|}{$\begin{array}{l}\text { Tukey HSD } \\
\text { Multiple Comparisons }\end{array}$} \\
\hline \multicolumn{3}{|c|}{ Dependent Variable: Time to Fusion } \\
\hline (I) Brand of stimulator & (J) Brand of stimulator & Sig. \\
\hline \multirow[t]{3}{*}{ No-Stim } & PEMF & 0.343 \\
\hline & $\mathrm{CMF}$ & 0.061 \\
\hline & $\mathrm{CC}$ & 0.685 \\
\hline \multirow[t]{3}{*}{ PEMF } & No-Stim & 0.343 \\
\hline & $\mathrm{CMF}$ & 0.000 \\
\hline & $\mathrm{CC}$ & 0.061 \\
\hline \multirow[t]{3}{*}{ CMF } & No-Stim & 0.061 \\
\hline & PEMF & 0.000 \\
\hline & $\mathrm{CC}$ & 0.771 \\
\hline \multirow[t]{3}{*}{ CC } & No-Stim & 0.685 \\
\hline & PEMF & 0.061 \\
\hline & CMF & 0.771 \\
\hline
\end{tabular}

CMF was significantly lower than No-Stim, $p=0.061 ; \mathrm{CMF}$ was significantly lower than PEMF, $p<0.001$.

not statistically significant (Table 7, Table 10 and Table 11).

The other patient subgroup studied for this outcome was overall smokers. For these patients, the CMF group had a mean fusion time of 175 days (SD 113.05), which was 67 days faster than the No-Stim group and the fastest among all patient groups. The mean fusion times for the CC, PEMF and No-Stim groups were 216 mean days (SD 128.38), 219 mean days (SD 185.52), and 242 mean days (SD 161.37), respectively, (Table 7).

\section{Adverse events}

In patients for which we could obtain complication data, it was found that the overall post-op complication rate was $15.5 \%(46 / 297)$. There were no significant differences in the occurrence of these events between any of the groups. According to the CMF SpinaLogic company, no known significant adverse events have resulted from the use of their BGS device in ether clinical or preclinical studies. None have been reported in the literature either. The same was found for the PEMF and CC technologies. Because of the latter and former, and the fact that the complication rates for all the patient cohorts hovered around $15.5 \%$, we have reason to believe that these complications occurred because of the surgery and are not attributable to the use of bone growth stimulators.

\section{Discussion}

We conducted this retrospective review to better understand the effectiveness of bone growth stimulators in promoting lumbar spinal fusion under different clinical scenarios. Use of any of the BGS technologies was safe and did not result in a significant increase in adverse events. When looking at the overall patient population, all of the BGS technologies (CMF, PEMF or CC) resulted in an increase in fusion rate when compared to no stimulation. This result is corroborated in the literature. A systematic review which examined the effect of electrical stimulation on lumbar fusions reported fusion rates following PEMF stimulation to be in the range of $64 \%$ to $83 \%$ while fusion rates following no stimulation ranged from $43 \%$ to $81 \%$. Similarly, the fusion rate following CC stimulation was $91 \%$, which was greater than the fusion rate of $82 \%$ following no stimulation [19].

A randomized prospective study completed by Linovitz [8] looked at the effect of CMF stimulation on fusion rate and reported a statistically significant $21 \%$ increase in fusion rates when using CMF stimulation compared to placebo. In our study, among patients receiving electrical stimulation, those receiving CMF stimulation had the highest fusion rates. While the Linovitz study showed CMF's superiority to no stimulation, and a study by El Hashemi [20] showed higher solid fusion rates for patients receiving CMF stimulation compared to PEMF stimulation, there is no current literature which shows a higher fusion rate following CMF stimulation when compared to CC stimulation.

Patients receiving any type of electrical stimulation also fused faster than patients receiving no stimulation. Our study is the first to show a decrease in fusion time following CMF stimulation when compared to PEMF stimulation and CC stimulation. The difference in fusion time was 75 days ( $p=$ 0.02 ) between the CMF and PEMF groups, 42 days between the CMF and CC groups, and 78 days between the CMF and No-Stim groups $(p=0.003)$. Although the Linovitz [8] study reported an accelerated time-to-heal for patients receiving CMF stimulation, the study only compared CMF patients to those receiving placebo.

In addition to having the highest fusion rates and shortest times-to-fusion among all cohorts for the overall patient population, the CMF group also had the highest fusion rates and shortest times-to-fusion for all patient subgroups. Smoking is an established spinal indication for the use of electrical bone growth stimulators [16]. It is a 
known high risk factor for lumbar spinal surgery and has been repeatedly shown to be associated with an increased risk of pseudoarthrosis and poor recovery [6]. Our results show that for patients with a smoking history, CMF stimulation resulted in the greatest fusion rate and fastest time-to fusion. It has been shown in the literature that PEMF stimulation can enhance smoker fusion rates [21] which held true in our study, but to date no comparison has been made in the literature between the BGS technologies or between CMF and no stimulation in regards to the fusion success of smokers.

Diagnosis is another patient-specific factor reported on in the literature in relation to bone growth stimulators' effect on fusion. Grade II+ spondylolisthesis is the only diagnosis shown in the literature to be a spinal indication for the use of electrical bone growth stimulators [16]. When investigating the relationship between various diagnoses and fusion success in the different patient cohorts, we discovered that for patients with a primary diagnosis of spinal stenosis, the CMF group had the highest fusion rate and fastest time-to-fusion.

In fact, spinal stenosis patients receiving CMF stimulation fused more than 200 days faster than patients receiving PEMF stimulation $(p<0.001)$ and more than 100 days faster than patients receiving no stimulation (not statistically significant). To our knowledge, no studies to date have discussed spinal stenosis as a possible indication for using a BGS. Given the fact that spinal stenosis is one of the most common indications for lumbar fusion surgery [1] and patients receiving fusion with this diagnosis have been shown to be at an increased risk of reoperation and complications [22], the potential for bone growth stimulators to promote fusion success in this subgroup has significant implications. Other patient factors investigated in this stu$d y$ in which there was data for all patient groups include gender, age and history of previous spine surgery.

As seen in the literature, use of electrical stimulation in the current study resulted in an increase in fusion rates for females but not males $[7,8]$. No comparisons are made among the BGS technologies in the literature. In our study, females receiving CMF stimulation had the greatest fusion rates compared to females receiving PEMF or CC stimulation. The CMF stimulator group also had the highest fusion rates among all patient groups for patients aged 61-70 and patients with a history of previous spine surgery, both of which are known risk factors for lumbar fusion surgery $[23,24]$. Literature could not be found on the effect of electrical bone growth stimulation on lumbar fusion success in these patient subgroups.

The current study demonstrates that electrical bone growth stimulation post-operatively results in an increase in fusion rate and a decrease in fusion time. The CMF BGS proved to be the most effective among the BGS technologies studied, both for the overall patient population and all stratification groups. It is important to recognize that the CMF stimulator has a much shorter wear-time than the PEMF or CC stimulator. Patients used the CMF stimulator for 30 minutes/day as opposed to 8 hours/day for the PEMF stimulator and 24 hours/day for the CC stimulator.
Wear time is important since the duration of commitment could put patient compliance in jeopardy. Without consistent schedule adherence, the benefits from BGS treatment may not be realized. A low maintenance device, such as the CMF SpinaLogic, requiring only 30 minutes a day makes the patient more apt to comply with the necessary home self-care.

Bone healing, or fusion, is the ultimate goal of spinal fusion procedures. A delay or failure in healing (pseudoarthrosis) can cause serious patient morbidity and often results in secondary procedures that are expensive and invasive, resulting in societal and personal costs such as postponements in returning to work, diminished productivity and lost wages [25]. To combat bone healing complications, there has been an increase in good surgical practices, knowledge and design which have improved fusion rates [16]. The use of BGSs for spinal fusions has been shown to be a cost-effective adjunctive treatment option [16], and a review of the literature indicates that treatment with $\mathrm{CMF}$ is clinically beneficial for lumbar spinal fusions, reducing the occurrence of pseudoarthrosis, and may be economically advantageous because of less time away from work and reduced risk of reoperation [1]. Spinal fusions and their recovery are a significant burden on patients, their caregivers, and the healthcare system. The benefits of electrical stimulation for certain populations such as the elderly, postmenopausal women, and smokers, all of who demonstrate a greater fusion response to CMF treatment [18], should not be overlooked.

Our findings are strengthened by the large scope of the study, the wide range of clinical eligibility criteria, the number of participating surgeons, and the comparative and comprehensive nature of the study. Limitations of our study include its retrospective nature, limited data availability, heterogeneity of the patient and physician populations, and the fact that fusions were not entirely determined by radiography.

\section{Conclusions}

Lumbar spine surgery demand in the U.S. will increase due to a rise in the aging population. Spinal surgery costs are increasing and with the constellation of care options available, the need to optimize treatment is more important now than ever. The data in this study shows that non invasive electrical bone growth stimulation is beneficial in promoting fusion success. We conclude that the integration of a BGS, specifically the CMF SpinaLogic BGS, in the aftercare healing of lumbar spinal fusion patients can potentially move the lumbar fusion field towards a higher expected fusion rate and a decreased time-to-fusion.

CMF stimulation was found to be the most effective bone growth stimulator technology among all those studied, as use of this stimulator resulted in the shortest fusion times and highest fusion rates. Given the short wear time of the CMF product, using it as an adjunct to lumbar spinal fusion surgery is very appealing. Choosing the right BGS is important for the wellbeing and quality of life of the patient.

These results provide a platform for future real-world evi- 
dence-based studies and help provide an example of the value of evidence-based decision making in the post-operative management of spinal fusion patients.

\section{Author Contributions}

Conceptualization: Rae Stegall and Rami Elsabeh; Methodology: Rae Stegall and Rami Elsabeh; Investigation: Rami Elsabeh and John Abrahams; Data Curation: Rae Stegall; Writing -Original Draft Preparation: Rae Stegall and Rami Elsabeh; Writing - Review and Editing: Rami Elsabeh and John Abrahams.

\section{Funding}

This research was funded by DJO Global.

\section{Acknowledgments}

Brain and Spine Surgeons of New York (BSSNY) includes 11 surgeons that contributed to the retrospective analysis. These surgeons are as follows: Dr. John Abrahams, Dr. Kaushik Das, Dr. Ezriel Kornel, Dr. Virany Hillard, Dr. Alain DeLotbiniere, Dr. Craig Shannon, Dr. Krishn Sharma, Dr. Rudolph Taddonio, Dr. Kent Duffy, Dr. Jack Stern, and Dr. Seth Neubardt. Data abstraction was completed by Qualitee 360, a healthcare data analytics firm.

\section{Conflicts of Interest}

Rami Elsabeh and John Abrahams declare no conflict of interest. Rae Stegall is a paid consultant for DJO Global.

\section{References}

1. Martin BI, Mirza SK, Spina N, et al. (2019) Trends in lumbar fusion procedure rates and associated hospital costs for degenerative spinal diseases in the united States, 2004 to 2015. Spine 44: 369-376.

2. Goz V, Weinreb JH, McCarthy I, et al. (2013) Perioperative complications and mortality after spinal fusions: Analysis of trends and risk factors. Spine 38: 1970-1976.

3. Baldwin P, Li DJ, Auston DA, et al. (2019) Autograft, Allograft, and Bone Graft Substitutes: Clinical evidence and indications for use in the setting of orthopaedic trauma surgery. J Orthop Trauma 33: 203-213.

4. Mobbs RJ, Phan K, Thayaparan GK, et al. (2016) Anterior lumbar interbody fusion as a salvage technique for pseudarthrosis following posterior lumbar fusion surgery. Global Spine J 6: 014-020.

5. Kornblum MB, Fischgrund JS, Herkowitz HN, et al. (2004) Degenerative lumbar spondylolisthesis with spinal stenosis: A prospective long-term study comparing fusion and pseudarthrosis. Spine 29: 726-733.

6. Berman D, Oren JH, Bendo J, et al. (2017) The effect of smoking on spinal fusion. Int J Spine Surg 11: 29-29.

7. Tian NF, Wu YS, Zhang XL, et al. (2013) Efficacy of electrical stimulation for spinal fusion: A meta-analysis of fusion rate. Spine $J$ 13: 1238-1243.

8. Linovitz RJ, Pathria M, Bernhardt M, et al. (2002) Combined magnetic fields accelerate and increase spine fusion: A double-blind, randomized, placebo controlled study. Spine 27: 1383-1389.
9. Nandyala SV, Marquez-Lara A, Fineberg SJ, et al. ( 2014) Prospective, Randomized, Controlled trial of silicate-substituted calcium phosphate versus rhBMP-2 in a minimally invasive transforaminal lumbar interbody fusion. Spine 39: 185-191.

10. Jenkins LT, Jones AL, Harms JJ (1994) Prognostic factors in lumbar spinal fusion. Contemp Orthop 29: 173-180.

11. Bydon M, De la Garza-Ramos R, Abt NB, et al. (2014) Impact of smoking on complication and pseudarthrosis rates after singleand 2-level posterolateral fusion of the lumbar spine. Spine 39: $1765-1770$.

12. Martin BI, Mirza SK, Comstock BA, et al. (2007) Reoperation rates following lumbar spine surgery and the influence of spinal fusion procedures. Spine 32: 382-387.

13. Chun DS, Baker KC, Hsu WK (2015) Lumbar pseudarthrosis: A review of current diagnosis and treatment. Neurosurg Focus 39: E10.

14. Miyazaki M, Tsumura H, Wang JC, et al. (2009) An update on bone substitutes for spinal fusion. Eur Spine J 18: 783-799.

15. Stippick TW, Sheller MR (2016) Combined magnetic fields provide robust coverage for interbody and posterolateral lumbar spinal fusion sites. Med Biol Eng Comput 54: 113-122.

16. Khalifeh JM, Zohny Z, MacEwan M, et al. (2018) Electrical stimulation and bone healing: A review of current technology and clinical applications. IEEE Reviews in Biomedical Engineering 11: 217-232.

17. Gan JC, Glazer PA (2006) Electrical stimulation therapies for spinal fusions: Current concepts. Eur Spine J 15: 1301-1311.

18. Phillips M, Drew B, Sprague S, et al. (2016) Efficacy of combined magnetic field treatment on spinal fusion: A review of the literature. J Long Term Eff Med Implants 26: 271-276.

19. Park P, Lau D, Brodt ED, et al. (2014) Electrical stimulation to enhance spinal fusion: A systematic review. Evid Based Spine Care J 5: 87-94.

20. EL Hashemi M, Khoi D Than, Barry Cheaney (2019) Combined magnetic field is superior to pulsed electromagnetic field bone stimulation after thoracolumbar fusion surgery. Joint Event on Neurology \& Brain Injury.

21. Marks RA (2000) Spine fusion for discogenic low back pain: Outcomes in patients treated with or without pulsed electromagnetic field stimulation. Advances in Therapy 17: 57-67.

22. Yavin D, Casha S, Wiebe S, et al. (2017) Lumbar fusion for degenerative disease: A systematic review and meta-analysis. Neurosurgery 80: 701-715.

23. Zhang L, Li EN (2018) Risk factors for surgical site infection following lumbar spinal surgery: A meta-analysis. Ther Clin Risk Manag 14: 2161-2169.

24. Schoenfeld AJ, Ochoa LM, Bader JO, et al. (2011) Risk factors for immediate postoperative complications and mortality following spine surgery: A study of 3475 patients from the national surgical quality improvement Program. J Bone Joint Surg Am 93: 1577-1582.

25. Aleem IS, Aleem I, Evaniew N, et al. (2016) Efficacy of electrical stimulators for bone healing: A meta-analysis of randomized sham-controlled trials. Sci Rep 6: 31724. 